Published By Institute of Physics Publishing for SiSSA/ISAS

RECEIVED:

REVISED:

ACCEPTED:

\title{
Partially composite two-Higgs doublet model
}

\author{
Byungchul Chung, Kang Young Lee \\ Department of Physics, KAIST, Daejeon 305-701, Korea \\ E-mail: crash@muon.kaist.ac.kr, kylee@muon.kaist.ac.kn

\section{Dong-Won Jung, Pyungwon Ko} \\ School of Physics, KIAS, Seoul 130-722, Korea \\ E-mail: dwjung@kias.re.kr, pko@kias.re.kr
}

\begin{abstract}
In the extra dimensional scenarios with gauge fields in the bulk, the KaluzaKlein (KK) gauge bosons can induce Nambu-Jona-Lasinio (NJL) type attractive fourfermion interactions, which can break electroweak symmetry dynamically with accompanying composite Higgs fields. We consider a possibility that electroweak symmetry breaking (EWSB) is triggered by both a fundamental Higgs and a composite Higgs arising in a dynamical symmetry breaking mechanism induced by a new strong dynamics. The resulting Higgs sector is a partially composite two-Higgs doublet model with specific boundary conditions on the coupling and mass parameters originating at a compositeness scale $\Lambda$. The phenomenology of this model is discussed including the collider phenomenology at LHC and ILC.
\end{abstract}

KEYWORDS: dynamical symmetry breaking, two-Higgs doublets model. 


\section{Contents}

1. Introduction 1

2. A Model of Dynamical Symmetry Breaking with a Fundamental Scalar

3. Particle Spectrum 6

4. Low Energy Phenomenology 10

4.1 Higgs boson self couplings 10

4.2 Signatures at the LHC 11

4.3 Signatures at the ILC

5. Conclusions 12

\section{Introduction}

Understanding the origin of electroweak symmetry breaking (EWSB) is one of the most important problems in particle physics. EWSB is strongly tied with the masses of chiral fermions and electroweak gauge bosons as well as $\mathrm{CP}$ violation in the standard model (SM) of Glashow-Salam-Weinberg. The nature of EWSB will be experimentally studied in detail at the CERN Large Hadron Collider (LHC) and the future $e^{+} e^{-}$linear collider (ILC). One may observe the force quanta, Higgs boson(s), that breaks electroweak symmetry in the standard model (SM), or its supersymmetric extensions. Or one may observe another signatures if there is no fundamental Higgs. It is an important phenomenological issue to extract maximal informations on the EWSB sector from the data obtainable at the LHC and the ILC. On the theoretical side, one has to study various models of EWSB including the SM which has a single Higgs doublet, compare with the data, and figure out which mechanism of EWSB is realized in nature.

In fact, there have been a lot of efforts to construct interesting models for the EWSB for more than the last two decades. In principle, studying EWSB is not necessarily related to solving the gauge hierarchy problem which is a fine tuning problem. But they are often interwined in actual model buildings. We would not care about solving the gauge hierarchy problem. Then there are a few different avenues to extend the SM regarding the EWSB:

- More fundamental Higgs : More fundamental Higgs singlets, doublets and triplets. This includes the general 2 Higgs doublet model, the minimal supersymmetric standard model (MSSM) and its various extensions [1]. In most cases, theories are weakly coupled and perturbation theory works. 
- Composite Higgs or dynamical EWSB : (extended) Technicolor scenarios, top condensate, topcolor, top seesaw, Little Higgs etc. [2, 3, 4, 5, 6]. In most cases, the EWSB sector is strongly coupled, and perturbation theory cannot be applied directly. One often has to construct effective field theory (EFT) to analyze the EWSB sector. There could be composite Higgs in the low energy spectra, but not necessariliy or always.

- Extra dimension : More options are avaiable in the extra dimensional scenarios. EWSB can arise either by fundamental Higgs, or by boundary conditions or by dynamical symmetry breaking from extra dimensional QCD [7, 8]. In fact, all the three options are generic in the extra dimensional scenarios and should be considered altogether in principle. This is in a sense similar to supersymmetry (SUSY) breaking scenarios, gravity mediation, gauge mediaion, anomaly mediation, gaugino mediation, etc., and some of these mediations could be comparable with each other.

Dynamical symmetry breaking á la Bardeen-Hill-Lindner (BHL) [3] is a particularly interesting scenario, since the heavy top mass is intimately related with a new strong dynamics that condenses the $t \bar{t}$ bilinear, and breaks the EW symmetry down to $U(1)_{\mathrm{EM}}$. Both heavy top mass and Higgs mass are generated dynamically, in anology with superconductivity of Bardeen-Cooper-Schrieffer (BCS) [9]. The original version of BHL with 3 families predicts that the top mass should be larger than $\sim 200 \mathrm{GeV}$, which is no longer viable considering the present measurement $m_{t}=178 \pm 4.3 \mathrm{GeV}$ [10]. Extension of BHL with two Higgs doublets has a similar shortcoming [11]. The top mass can be lowered to the observed value, if one considers supersymmetric extension 12] or if there is the 4-th generation [1]. Another conceptual problem of BHL scenario is the origin of the new strong interactions that triggers electroweak symmetry breaking. The attractive 4 -fermion interaction is simply put in by hand within the BHL model. Despite these drawbacks, we believe that it is worthwhile to consider variations of the BHL scenario, since these two drawbacks can be easily evaded within extra dimensional scenarios, without ruining its niceties: this scenario is attractive, could be generic, and provides dynamical origins for fermion and gauge boson masses (at least a part of them) in the extra dimensional scenarios.

A new trend in model building for the last decade was to use extra dimesions to solve gauge hierarchy problem, and/or fermion mass hierarchy [13]. If QCD is a bulk theory, then it is possible that the extra dimensional QCD can induce attractive Nambu-Jona-Lasinio (NJL) type four-fermion interaction in the low energy regime, and dynamical symmetry breaking can occur as usual. In short, one has a natural setting for dynamical symmetry breaking from extra dimensional QCD. It should be emphasized that this is completely different from another popular way of symmetry breaking in extra dimension, namely symmetry breaking by boundary conditions. We believe that it is not an option but an obligation to study the dynamical EWSB in extra dimensional scenarios, if once we have gauge theories in the bulk. After the large extra dimension scenario was put forward, a few groups studied dynamical symmetry breaking in flat extra dimensions [14 and in warped extra dimension [15]. The qualitative result from these studies is that it is indeed possible to have dynamical EWSB from extra dimensional QCD. Then in extra dimensional scenarios, 
electroweak symmetry can be broken by fundamental Higgs, by boundary condition or by some dynamical mechanism. Generically all three possibilities could be present altogether. In recent literatures, each option of EWSB in extra dimensional scenarios was discussed in detail. In most cases, the gauge symmetries are broken by both fundamental Higgs as well as the nontrivial boundary conditions, or completely by boundary conditions without Higgs. Another interesting possibility that electroweak symmetry is broken by fundamental Higgs VEV's, and dynamically by $t \bar{t}$ as well. condensate, has not been considered yet to our best knowledge. It is the purpose of this work to consider this possibility in a minimal setup.

In this paper, we consider an extension of BHL scenario, where one has a fundamental Higgs from the beginning, and the $t \bar{t}$ bilinear condensate due to a new strong interaction which is triggered by extra dimensional QCD. We assume this is achieved by embedding the $\mathrm{SM}$ in the higher dimensional spacetime with an appropriate extra dimensional QCD. Since we have both fundamental and composite Higgs fields, it is natural that the low-energy effective theory is the two-Higgs doublet model. If we assume that the fundamental Higgs couples only to the bottom quark and the top quark purely receives its large mass from dynamical EWSB as in BHL, then the resulting low energy effective theory is a Type-II two-Higgs doublet model with one fundamental and one composite Higgs doublets. In our model, the top mass can be fit to the observed value for the limited range of $\tan \beta$. Actually only a narrow window for $0.45 \lesssim \tan \beta \lesssim 1$ is allowed in our model for a given compositeness scale $\Lambda$. For such a $\tan \beta$, roughly half of the $W$ and $Z$ masses come from the fundamental Higgs and half from the dynamical symmetry breaking. Since the phyical Higgs bosons are linear combinations of fundamental and composite Higgs bosons, we call it the partially composite Higgs boson. In a sense, our model is somewhere between the general two Higgs doublet model and the composite two Higgs doublet model by Luty [11]. Compared with the model by Luty, we have one more parameter, the Yukawa coupling between the fundamental Higgs $\phi$ and the bottom quark at the compositeness scale. Then we can fit the heavy top mass without trouble unlike the Luty model. So the phenomenological disaster of the BHL-like top condensation model is gone in our model.

Both in the BHL model and our model, the fine tuning problem of the Higgs mass is not explained. Unlike the general two Higgs doublet model, this model has only two more free parameters compared with the SM: the CP-odd neutral Higgs mass $m_{A}$ and the composite scale $\Lambda$. (The quartic self-coupling $\lambda_{10}$ in our model is a free parameter as in the SM.) Therefore our model is more predictable and testable than the general two-Higgs doublet models, and could be possibly verified or excluded in the future colliders.

Although our work is motivated by the extra dimensional scenarios, the model presented in this paper is not a genuine model that would be obtained when we embed the SM in the higher dimensional world. Generically we would have Type-III general two-Higgs doublet models if only the top quark couples to the dynamical symmetry breaking sector. If the bottom quarks also couple to the dynamicall symmetry breaking sector, then the resulting low energy effective theory would be a three-Higgs doublet model. In this work, we assume that it is the top quark which feels the dynamical EWSB sector, and we will introduce a discrete symmetry in order to reduce the Type-III Higgs doublet model to the 
Type-II Higgs doublet model. This will help to suppress dangerous Higgs mediated flavor changing neutral current processes. And the top mass is generated entirely from dynamical EWSB. It is beyond the scope of the current paper to discuss more general and realistic models with 3 generations of fermions into account, since it requires a more involved RG analysis for general two Higgs doublet model. This issue will be addressed in the future publication [16].

This paper is organized as follows. In Section 2, we define our model extending the BHL scenario. In Section 3, we present the mass spectra and the couplings of two-Higgs doublets in our model. In Section 4, we consider the Higgs boson productions at ILC and LHC, and study the discoversy potential therein. We use the one-loop RG equations for couplings in the effective theory of our model, which are collected in Appendix for convenience.

\section{A Model of Dynamical Symmetry Breaking with a Fundamental Scalar}

We introduce a strong dynamics to the standard model at some high scale $\Lambda$, which is effectively described by the NJL type four-fermion interaction term. Although we don't have to specify the origin of this NJL type interaction, we have in mind the KK gauge boson exchange as the origin of this new strong interaction as discussed in the Introduction. As a minimal extension of the SM, we assume that this new strong dynamics acts only on top quark. Then we can write the lagrangian at the scale $\Lambda$ as:

$$
\mathcal{L}=\mathcal{L}_{\mathrm{SM}}+G\left(\bar{\psi}_{L} t_{R}\right)\left(\bar{t}_{R} \psi_{L}\right)
$$

where

$$
\mathcal{L}_{\mathrm{SM}}=\mathcal{L}_{\text {gauge }}+\mathcal{L}_{f}+\mathcal{L}_{\phi}+\left(y_{t 0} \bar{\psi}_{L} t_{R} \tilde{\phi}+\text { H.c. }\right)+\left(y_{b 0} \bar{\psi}_{L} b_{R} \phi+\text { H.c. }\right)
$$

and $\tilde{\phi} \equiv i \sigma_{y} \phi^{*}$. The fermion and scalar field lagrangians are gauge invariant kinetic terms, given by

$$
\begin{aligned}
& \mathcal{L}_{f}=\bar{\psi}_{L}^{a} i \not D \psi_{L}^{a}+\bar{q}_{R}^{a} i \not D q_{R}^{a}, \\
& \mathcal{L}_{\phi}=\left(D_{\mu} \phi\right)^{\dagger}\left(D^{\mu} \phi\right)-V(\phi),
\end{aligned}
$$

where $a$ is the generation index, $\psi_{L}$ is the $\mathrm{SU}(2)_{\mathrm{L}}$ doublet and $q_{R}$ the $\mathrm{SU}(2)_{\mathrm{L}}$ singlet. The scalar potential is given by

$$
V(\phi)=m_{0}^{2} \phi^{\dagger} \phi+\frac{1}{2} \lambda_{0}\left(\phi^{\dagger} \phi\right)^{2}
$$

as in the SM. $y_{t 0}$ and $y_{b 0}$ are Yukawa couplings of the top and the bottom quarks to the fundamental Higgs field $\phi$. The Yukawa couplings for the 1st and the 2nd generations do not play any roles in our analysis, and will be ignored in the following.

Introducing an auxiliary scalar doublet field $\Phi(x)$, we can rewrite the NJL term in Eq. (2.1) as

$$
\mathcal{L}=\mathcal{L}_{\mathrm{SM}}+g_{t 0}\left(\bar{\psi}_{L} t_{R} \tilde{\Phi}+\text { H.c. }\right)-M^{2} \Phi^{\dagger} \Phi
$$


where $G=g_{t 0}^{2} / M^{2}$ and $g_{t 0}$ is a newly defined Yukawa coupling between the top quark and the auxiliary scalar field $\Phi$. The mass scale $M$ will be generically of order $\Lambda$. The new scalar field $\Phi$ describes the composite scalar bosons that appear when the $\langle\bar{t} t\rangle$ develops nonvanishing VEV and breaks EWSB by NJL type new strong dynamics. Then we have one fundamental scalar field $\phi$ and one composite scalar field $\Phi$, although $\Phi$ is not a dynamical field at the scale $\Lambda$. Far below the scale $\Lambda$, the $\Phi$ field will develop the kinetic term due to quantum corrections and become dynamical. The resulting low energy effective field theory will be two-Higgs doublet model, one being a fundamental Higgs $\phi$ and the other being a composite Higgs $\Phi$. Thus it can be called a partially composite two-Higgs doublet (PC2HD) model. In a general two-Higgs doublet model, one may have too excessive FCNC amplitudes mediated by neutral Higgs bosons. This phenomenological problem can be avoided if one invokes the Glashow-Weinberg criteria [17]. For simplicity, we assign a $Z_{2}$ discrete symmetry under which the lagrangian is invariant;

$$
\begin{aligned}
\left(\Phi, \psi_{L}, U_{R}\right) & \rightarrow+\left(\Phi, \psi_{L}, U_{R}\right), \\
\left(\phi, D_{R}\right) & \rightarrow-\left(\phi, D_{R}\right) .
\end{aligned}
$$

With this $Z_{2}$ discrete symmetry, the Yukawa term $\left(y_{t 0} \bar{\psi}_{L} t_{R} \phi+\right.$ H.c. $)$ is forbidden in the lagrangian of Eq. (2.2), and only the $y_{b 0}$ coupling term remains ${ }^{1}$. In consequence, our model becomes the Type-II two-Higgs doublet model, where the fundamental scalar field $\phi$ couples to the down-type quarks, and the composite scalar $\Phi$ couples to the up-type quarks. If one allows the fundamental Higgs to couple to the top quark, the top mass will get contributions both from the $\langle\phi\rangle$ and $\langle\Phi\rangle$, and the resulting low energy effective theory will be a Type-III two-Higgs doublet model. Since the RG equations for scalar self-couplings are not known in general Type-III models, we postpone discussing this case for the future study [16].

Therefore the Higgs structure of our model is the same as that of the MSSM. It has a virtue of the natural suppression of the FCNC and is the most popular two-Higgs model. We write the effective lagrangian far below $\Lambda$ as

$$
\begin{aligned}
\mathcal{L}=\mathcal{L}_{\text {gauge }} & +\mathcal{L}_{f}+\left(D_{\mu} \phi\right)^{\dagger}\left(D^{\mu} \phi\right)+\left(D_{\mu} \Phi\right)^{\dagger}\left(D^{\mu} \Phi\right) \\
+ & \left(g_{b} \bar{\psi}_{L} b_{R} \phi+\text { H.c }\right)+\left(g_{t} \bar{\psi}_{L} t_{R} \tilde{\Phi}+\text { H.c }\right)-V(\phi, \Phi),
\end{aligned}
$$

where the most general Higgs potential is given by

$$
\begin{aligned}
V(\phi, \Phi)= & \mu_{1}^{2} \phi^{\dagger} \phi+\mu_{2}^{2} \Phi^{\dagger} \Phi+\left(\mu_{12}^{2} \phi^{\dagger} \Phi+\text { H.c. }\right) \\
& +\frac{1}{2} \lambda_{1}\left(\phi^{\dagger} \phi\right)^{2}+\frac{1}{2} \lambda_{2}\left(\Phi^{\dagger} \Phi\right)^{2} \\
& +\lambda_{3}\left(\phi^{\dagger} \phi\right)\left(\Phi^{\dagger} \Phi\right)+\lambda_{4}\left|\phi^{\dagger} \Phi\right|^{2}+\frac{1}{2}\left[\lambda_{5}\left(\phi^{\dagger} \Phi\right)^{2}+\text { H.c. }\right] .
\end{aligned}
$$

In the scalar potential, we have introduced a dimension-two $\mu_{12}^{2}$ term that breaks the discrete symmetry softly in order to generate the nonzero mass for the CP-odd Higgs boson.

\footnotetext{
${ }^{1}$ From now on, we will rename $y_{b 0}$ as $g_{b 0}$.
} 
Otherwise the CP-odd Higgs boson $A$ would be an unwanted axion related with spontanesously broken global $U(1)$ Peccei-Quinn symmetry, which would be a phenomenological disaster. This $\mu_{12}^{2}$ term will not induce dangerously large FCNC amplitudes at loop levels, thus not spoiling our original motivation to consider the discrete symmetry [1]. In the next section, this $\mu_{12}^{2}$ parameter will be traded with the $m_{A}^{2}$, the (mass) ${ }^{2}$ parameter of the CP-odd Higgs boson, which is another free parameter of our model.

The renormalized lagrangian for the scalar fields at low energy is given by

$$
\begin{gathered}
\mathcal{L}_{\text {ren }}=Z_{\phi}\left(D_{\mu} \phi\right)^{\dagger}\left(D^{\mu} \phi\right)+Z_{\Phi}\left(D_{\mu} \Phi\right)^{\dagger}\left(D^{\mu} \Phi\right)-V\left(\sqrt{Z_{\phi}} \phi, \sqrt{Z_{\Phi}} \Phi\right) \\
+\sqrt{Z_{\Phi}} g_{t}\left(\bar{\psi}_{L} t_{R} \tilde{\Phi}+\text { h.c }\right)+\sqrt{Z_{\phi}} g_{b}\left(\bar{\psi}_{L} b_{R} \phi+\text { h.c }\right),
\end{gathered}
$$

and matching the lagrangian with Eq. (2.5) at the compositeness scale $\Lambda$, we obtain the matching condition

$$
\begin{aligned}
& \sqrt{Z_{\phi}} \rightarrow 1, \quad \sqrt{Z_{\Phi}} \rightarrow 0, \\
& Z_{\phi} \mu_{1}^{2} \rightarrow m_{0}^{2}, \quad Z_{\Phi} \mu_{2}^{2} \rightarrow M^{2}, \\
& Z_{\phi} \lambda_{1} \rightarrow \lambda_{10}, \quad Z_{\Phi}^{2} \lambda_{2} \rightarrow 0, \\
& Z_{\phi} Z_{\Phi} \lambda_{i=3,4,5} \rightarrow 0,
\end{aligned}
$$

as the scale $\mu \rightarrow \Lambda$. Now the low energy theory is the Type-II two-Higgs doublet model with two Higgs fields $\phi$ and $\Phi$ with the compositeness conditions for $\Phi$ : $\Phi$ has a vanishing wavefunction renormalization constant at the compositeness scale $\Lambda$, while $\phi$ does not.

Before proceeding, we would like to compare our model with Luty's model, since both models are two-Higgs doublet models in the low energy regime. In Luty's model, both Higgs doublets are composite, and thus the matching conditions are given by

$$
\begin{aligned}
& \sqrt{Z_{\phi}} \rightarrow 0, \quad \sqrt{Z_{\Phi}} \rightarrow 0, \\
& Z_{\phi} \mu_{1}^{2} \rightarrow m_{0}^{2}, \quad Z_{\Phi} \mu_{2}^{2} \rightarrow M^{2}, \\
& Z_{\phi} \lambda_{1} \rightarrow 0, \quad Z_{\Phi}^{2} \lambda_{2} \rightarrow 0, \\
& Z_{\phi} Z_{\Phi} \lambda_{i=3,4,5} \rightarrow 0 .
\end{aligned}
$$

Namely the conditions for the scalar field $\phi$, the self coupling $\lambda_{1}$ and the wavefunction renormalization constant $Z_{\phi}$, are different from our case. These different matching conditions lead to vastly different predictions for the scalar boson spectra compared to the Luty's model. Also we have additional Yukawa coupling $g_{b}$ so that we can fit both the bottom and the top quark masses without difficulty unlike Luty's model.

\section{Particle Spectrum}

Our model is defined in terms of three parameters: Higgs self coupling $\lambda_{10}$, the compositeness scale $\Lambda$ (where $\lambda_{10}$ and the NJL interaction are specified), and the CP-odd Higgs boson mass $m_{A}$. Since $\lambda_{10}$ is also present in the SM, our model has two more parameters compared with the SM. In order to study the low energy phenomenology of our model, we 


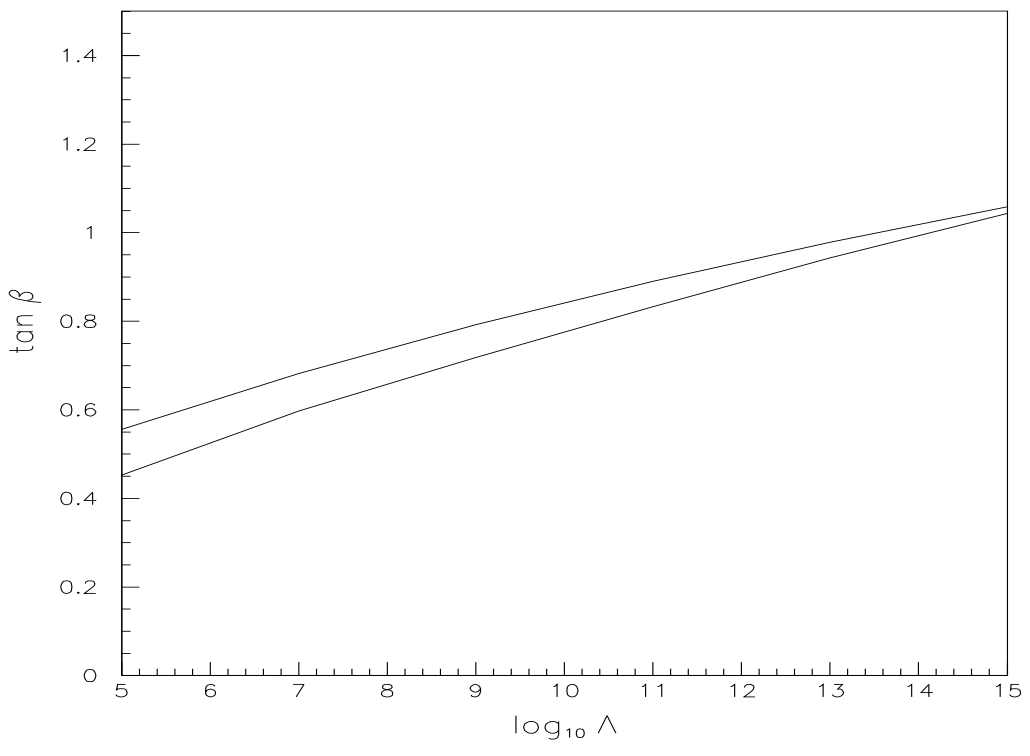

Figure 1: Allowed values of $\tan \beta$ with respect to the compositeness scale $\Lambda$.

relate the model defined at the high scale $\Lambda$ to the low-energy spectrum of the theory by evolving the renormalization group ( $\mathrm{RG}$ ) equation from $\Lambda$ to the electroweak scale with the compositeness conditions Eq. (2.9). Using the field redefinition

$$
\phi \rightarrow Z_{\phi}^{-1 / 2} \phi, \quad \Phi \rightarrow Z_{\Phi}^{-1 / 2} \Phi
$$

we rewrite the matching condition given in Eq. (2.9) as

$$
\begin{aligned}
& g_{b} \rightarrow g_{b 0}, \quad g_{t} \rightarrow \infty, \\
& \lambda_{1} / g_{b}^{4} \rightarrow \lambda_{10} / g_{b 0}^{4}, \quad \lambda_{2,3,4,5} \rightarrow 0,
\end{aligned}
$$

for the rescaled couplings. These conditions are the boundary conditions for the RG equations. The condition $g_{t} \rightarrow \infty$ and $\lambda_{2,3,4,5} \rightarrow 0$ at the compositeness scale $\Lambda$ are the compositeness condition for $\Phi$. No conditions are assigned on $g_{b 0}$ and $\lambda_{10}$ at this stage. They will be fixed or constrained by the phenomenological conditions and the electroweak symmetry breaking conditions. We will use the one-loop RG equations given in Ref. [18], which are reproduced in the Appendix for convenience.

We set the Higgs vacuum expectation values (VEVs) to be

$$
\langle\phi\rangle=\frac{1}{\sqrt{2}}\left(\begin{array}{c}
0 \\
v_{1}
\end{array}\right), \quad\langle\Phi\rangle=\frac{1}{\sqrt{2}}\left(\begin{array}{c}
0 \\
v_{2} e^{i \delta}
\end{array}\right),
$$


with the electroweak symmetry breaking scale $v^{2}=v_{1}^{2}+v_{2}^{2}=(246 \mathrm{GeV})^{2}$. We define $\tan \beta \equiv v_{2} / v_{1}$. The relative phase $\delta$ can be absorbed by an appropriate field redefinition, and we will drop it hereafter. The top and bottom quark masses are generated by the VEVs of $\phi$ and $\Phi$ :

$$
\begin{aligned}
& m_{t}=\frac{1}{\sqrt{2}} g_{t} v_{2}=\frac{1}{\sqrt{2}} g_{t} v \sin \beta, \\
& m_{b}=\frac{1}{\sqrt{2}} g_{b} v_{1}=\frac{1}{\sqrt{2}} g_{b} v \cos \beta .
\end{aligned}
$$

Since the boundary condition for $g_{t}$ is fixed to be the compositeness condition at $\Lambda, \tan \beta$ is almost fixed in our model. In the actual numerical analysis, we take the boundary condition of $g_{t}$ to be finite instead of infinity, but large enough for being in nonperturbative region, following the approaches of BHL and Luty. This approach is supported by the fact that the low-energy behavior of $g_{t}$ is not that sensitive to the boundary condition at $\Lambda$ when $g_{t} \rightarrow \infty$ because of the infrared fixed point behavior [11, 18]. Once $\tan \beta$ is fixed, the bottom Yukawa coupling $g_{b 0}$ at the composite scale is also determined by the measured bottom quark mass. We use the following values for the top and bottom quark masses 19.

$$
m_{t}\left(M_{Z}\right)=178.1 \mathrm{GeV}, \quad m_{b}\left(M_{Z}\right)=2.8 \mathrm{GeV},
$$

and gauge couplings as

$$
\alpha\left(M_{Z}\right)=\frac{1}{127.934}, \quad \alpha_{s}\left(M_{Z}\right)=0.1172, \quad \sin ^{2} \theta_{W}\left(M_{Z}\right)=0.2221 .
$$

As a result, only a very narrow window of $\tan \beta$ is consistent with the measured top and bottom quark masses for a given compositeness scale $\Lambda$. In Fig. 1, we show the allowed range of $\tan \beta$ for the compositeness scale $\Lambda$.

Evolutions of quartic couplings $\lambda_{i}$ are constrained by the stability and electroweak symmetry breaking conditions of the Higgs potential. Since the potential should to be bounded from below, we require that

$$
\begin{array}{ll}
\lambda_{1}, \lambda_{2}>0 & \\
\sqrt{\lambda_{1} \lambda_{2}}>-\lambda_{3}-\lambda_{4}+\left|\lambda_{5}\right| & \text { if } \lambda_{4}<\left|\lambda_{5}\right|, \\
\sqrt{\lambda_{1} \lambda_{2}}>-\lambda_{3} & \text { if } \lambda_{4}>\left|\lambda_{5}\right| .
\end{array}
$$

Minimizing the Higgs potential at $v_{1}$ and $v_{2}$, we obtain the following conditions for the electroweak symmetry breaking :

$$
\begin{aligned}
& \mu_{1}^{2}+\mu_{12}^{2} \tan \beta+\frac{1}{2} \lambda_{1} v_{1}^{2}+\frac{1}{2} \lambda_{345} v_{2}^{2}=0 \\
& \mu_{2}^{2}+\mu_{12}^{2} \cot \beta+\frac{1}{2} \lambda_{2} v_{2}^{2}+\frac{1}{2} \lambda_{345} v_{1}^{2}=0
\end{aligned}
$$

where $\lambda_{345}=\lambda_{3}+\lambda_{4}+\lambda_{5}$. The boundary condition for $\lambda_{1}, \lambda_{10} \equiv \lambda_{1}(\Lambda)$, is chosen so that the evolved couplings satisfy those conditions (3.6) and (3.7) at the electroweak scale. Typical evolutions of quartic couplings for two different $\lambda_{10}$ are shown in the Fig. 2 (a) and 
(b). Since $\lambda_{1}$ decreases almost monotonically, it becomes negative at the electroweak scale in most region of the parameter space when $\Lambda>10^{12} \mathrm{GeV}$ if $\lambda_{10}$ is small or moderate [ Fig. 2 (a) ]. For larger $\lambda_{10}$, the RG evolved $\lambda_{1}$ could be positive at electroweak scale [ Fig. 2 (b) ]. In such a case, the scalar self coupling $\lambda_{1,2}$ can be large so that the resulting triple or quartic Higgs self coulings could be enhanced significantly compared to the SM case. After all, the allowed parameter space for $\lambda_{10}$ is significantly reduced for large compositeness scale $\Lambda$. The allowed region of $\lambda_{10}$ is shown in Fig. 3 for different compositeness scale $\Lambda$.

The coupling $\lambda_{5}$ is very interesting, since it can contribute to the CP violation in the Higgs sector through mixing, if $\operatorname{Im}\left(\lambda_{5}\right) \neq 0$. It turns out that the one-loop beta function for $\lambda_{5}$ is proportional to $\lambda_{5}$ itself [ see Eq. (5.4)]. Since we have the vanishing initial condition for $\lambda_{5}=0$, we have $\lambda_{5}=0$ at all the scale down to $M_{Z}$. The $\mu_{12}^{2}$ term can also contribute to the $\mathrm{CP}$ violation in the Higgs sector if it has a $\mathrm{CP}$ violating phase. However the minimization condition for the scalar potential leads to 20]

$$
\operatorname{Im}\left(\mu_{12}^{2}\right)=-\frac{1}{2} \operatorname{Im}\left(\lambda_{5}\right) v_{1} v_{2}
$$

which can be obtained from the imaginary part of (3.6). Since $\lambda_{5}=0$ in our model, we have $\operatorname{Im}\left(\mu_{12}^{2}\right)=0$ and no Higgs sector $\mathrm{CP}$ violation from the phase of $\mu_{12}^{2}$ term in our model. This gurantees that there will be no mixing between the CP-even and the CP-odd neutral Higgs bosons. Finally, we will demand that the perturbativity condition $\lambda<4 \pi$ be satisfied in this analysis.

After EWSB, we have three neutral scalar fields and a couple of charged scalar. The mass matrix for real part of the neutral scalar fields $(\operatorname{Re} \phi, \operatorname{Re} \Phi)$ is given by

$$
M^{2}=\left(\begin{array}{cc}
\mu_{1}^{2}+\frac{3}{2} \lambda_{1} v_{1}^{2}+\frac{1}{2} v_{1} v_{2} \lambda_{345} & \mu_{12}^{2}+v_{1} v_{2} \lambda_{345} \\
\mu_{12}^{2}+v_{1} v_{2} \lambda_{345} & \mu_{2}^{2}+\frac{3}{2} \lambda_{2} v_{2}^{2}+\frac{1}{2} v_{1} v_{2} \lambda_{345}
\end{array}\right) .
$$

Diagonalizing the mass matrix, the physical CP-even Higgs bosons are defined by

$$
\begin{aligned}
H & =\sqrt{2}(\operatorname{Re} \Phi \sin \alpha+\operatorname{Re} \phi \cos \alpha), \\
h & =\sqrt{2}(-\operatorname{Re} \Phi \cos \alpha+\operatorname{Re} \phi \sin \alpha),
\end{aligned}
$$

with the mixing angle $\alpha$ defined by

$$
\tan 2 \alpha \equiv \frac{2 M_{12}^{2}}{M_{11}^{2}-M_{22}^{2}}
$$

and their masses are given by

$$
m_{H, h}=\frac{1}{2}\left[M_{11}^{2}+M_{22}^{2} \pm \sqrt{\left(M_{11}^{2}-M_{22}^{2}\right)^{2}+4 M_{12}^{2}}\right] .
$$

The CP-odd Higgs boson $A$ is defined by the orthogonal state of the pseudoscalar Goldstone mode, $A=\sqrt{2}(-\operatorname{Im} \Phi \sin \beta+\operatorname{Im} \phi \cos \beta)$ and its mass is given by

$$
m_{A}^{2}=-\frac{2 \mu_{12}^{2}}{\sin 2 \beta}-\lambda_{5} v^{2}
$$


Since $\lambda_{5}=0$ in our model, the mass of the CP-odd Higgs boson $A$ is generated solely by the soft breaking term $\mu_{12}^{2}$ whch is another free parameter in our model. Therefore, we take the mass of the CP-odd Higgs boson $m_{A}$ to be an input parameter in our analysis, traded with $\mu_{12}^{2}$, as mentionewd earlier.

Finally, the charged Higgs boson masses are given by

$$
m_{H^{ \pm}}^{2}=m_{A}^{2}-\frac{1}{2}\left(\lambda_{4}-\lambda_{5}\right) v^{2}=m_{A}^{2}-\frac{1}{2} \lambda_{4} v^{2} .
$$

The masses of neutral and charged Higgs bosons with varying $m_{A}$ are given in Fig. 4 . The vertical line denotes the mass bound of $A$ derived from the bound on $m_{H^{ \pm}}$as given in PDG. Generically experimental bound on $m_{H^{ \pm}}$is more stringent than that on $m_{A}$. We note that $\lambda_{4}$ becomes positive at the electroweak scale in most cases unlike Luty's model, since the contributions of $\lambda_{1}$ and $\lambda_{2}$ to the evolution is substantial. Consequently, the charged Higgs boson $H^{ \pm}$is generically lighter than the CP-odd Higgs boson in our model. As shown in Fig. 4, moreover, $H^{ \pm}$may be even lighter than the lightest neutral Higgs boson when the compositeness scale $\Lambda$ is high. This is a generic feature of our model.

\section{Low Energy Phenomenology}

\subsection{Higgs boson self couplings}

The Higgs boson self-couplings may play a role of the sensitive probe to the new physics and have great phenomenological significance [21, 22]. The ratios of the triple and the quartic self-couplings of the lighter CP-even Higgs boson $h$ in general two Higgs doublet model to those of the SM are given by [22] The ratio of self-couplings to the SM values are given by

$$
\begin{aligned}
\frac{\lambda_{h h h}}{\lambda_{h h h}^{\mathrm{SM}}} & =\frac{1}{4 \sqrt{2}}\left(-\frac{\lambda_{1}}{\lambda_{\mathrm{SM}}} \cos \beta \sin ^{3} \alpha+\frac{\lambda_{2}}{\lambda_{\mathrm{SM}}} \sin \beta \cos ^{3} \alpha-\frac{\lambda_{345}}{\lambda_{\mathrm{SM}}} \cos \alpha \sin \alpha \cos (\alpha+\beta)\right), \\
\frac{\lambda_{h h h h}}{\lambda_{h h h h}^{\mathrm{SM}}} & =\frac{1}{8}\left(\frac{\lambda_{1}}{\lambda_{\mathrm{SM}}} \sin ^{4} \alpha+\frac{\lambda_{2}}{\lambda_{\mathrm{SM}}} \cos ^{4} \alpha+2 \frac{\lambda_{345}}{\lambda_{\mathrm{SM}}} \cos ^{2} \alpha \sin ^{2} \alpha\right),
\end{aligned}
$$

where $\alpha$ is the mixing angle defined in (3.9) and (3.10). When $\tan \alpha$ tends to be very large, $\cos \alpha \ll \sin \alpha$ and the first terms of the above equations dominate in most parameter space. Thus $\lambda_{1} / \lambda_{\text {SM }}$ plays the crucial role for the ratio of self-couplings. And this could be large if the initial $\lambda_{10}$ is large, as shown in Fig. 2 (b). In Fig. 5 and Fig. 6, we show $\lambda_{h h h} / \lambda_{h h h}^{\mathrm{SM}}$ and $\lambda_{h h h h} / \lambda_{h h h h}^{\mathrm{SM}}$ as functions of $m_{h}$. We find that the deviations of the self-couplings from the SM values can be substantial, even close to ten times, when the CP-even Higgs boson $h$ becomes light. For the triple self-coupling, such an enhancement arises when $m_{A}>m_{h}$ in the small $m_{h}$ region through the squared mass ratio $m_{A}^{2} / m_{h}^{2} \sim 10$ in the second term of Eq. (4.1). Thus our triple self-coupling is negative in most parameter region, which can lead to many interesting phenomenologies due to the destructive interference with the SM contribution. For the quartic self-coupling, the ratio of $m_{H}^{2} / m_{h}^{2}$ is responsible for the enhancement since $m_{H}>m_{A}$ in most region of parameter space. These large deviations of Higgs self couplings from the SM predictions are the most interesting features of our model 
with one fundamental and one composite Higgs fields. The Higgs pair and the triple Higgs production cross sections at the ILC will be interesting signatures of our model, and the detailed phenomenology will be discussed elsewhere [16].

\subsection{Signatures at the LHC}

The main production mechanism for the lighter neutral CP-even Higgs boson $h$ at the LHC is the gluon-gluon fusion process, $g g \rightarrow h$. This process involves the top quark loop both in the SM and in our model since $\tan \beta \lesssim O(1)$, and the production cross section is determined by the Yukawa coupling. Since the Yukawa couplings in our model are the same as in the SM, the Higgs production at LHC will be the same as in the SM, and this process is not a good probe of our model. However, there are more Higgs bosons in our model compared with the SM. The CP-odd Higgs boson $A$ can be lighter than the CP-even Higgs (see Fig. 4) and then it is possible that the first observed Higgs would not be the CP-even $h$ and but the CP-odd $A$. Moreover, the charged Higgs boson $H^{ \pm}$can be lighter than $h$ (see Fig. 4). When the compositeness scale $\Lambda$ is high enough and $m_{h}<350 \mathrm{GeV}$, $m_{h}$ is always larger than $m_{H^{ \pm}}$. In that case, the charged Higgs could be observed before the neutral Higgs boson through the $g b$ fusion.

\subsection{Signatures at the ILC}

The future $e^{+} e^{-}$international linear collider (ILC) will examine the detailed structure of the Higgs sector (or EWSB sector). The most important channel for the neutral Higgs boson production at the ILC are the Higgs-strahlung process $\left(e^{-} e^{+} \rightarrow Z H\right)$ and the $W W$ fusion process $\left(e^{-} e^{+} \rightarrow W^{*} W^{*} \rightarrow \bar{\nu}_{e} \nu_{e} H\right.$ ) [23] where $H$ is one of the CP-even Higgs bosons $h^{0}, H^{0}$. The cross sections for these processes are expressed by

$$
\begin{aligned}
\sigma\left(e^{+} e^{-} \rightarrow Z+h^{0} / H^{0}\right) & =\sin ^{2} / \cos ^{2}(\beta-\alpha) \sigma_{\mathrm{SM}}\left(e^{+} e^{-} \rightarrow Z+h^{0} / H^{0}\right), \\
\sigma\left(e^{-} e^{+} \rightarrow \bar{\nu}_{e} \nu_{e}+h^{0} / H^{0}\right) & =\sin ^{2} / \cos ^{2}(\beta-\alpha) \sigma_{\mathrm{SM}}\left(e^{-} e^{+} \rightarrow \bar{\nu}_{e} \nu_{e}+h^{0} / H^{0}\right) .
\end{aligned}
$$

The SM cross section $\sigma_{\mathrm{SM}}$ is given by 23]

$$
\begin{aligned}
\sigma_{\mathrm{SM}}\left(e^{-} e^{+} \rightarrow Z+H\right) & =\frac{g_{Z Z H}^{2}}{4 \pi} \frac{G_{F}\left(v_{e}^{2}+a_{e}^{2}\right)}{96 \sqrt{2} s} \beta_{h} \frac{\beta_{h}^{2}+12 m_{Z}^{2} / s}{\left(1-m_{Z}^{2} / s\right)^{2}} \\
\sigma_{\mathrm{SM}}\left(e^{-} e^{+} \rightarrow \bar{\nu}_{e} \nu_{e}+H\right) & =\frac{g_{W W H}^{2}}{4 \pi} \frac{G_{F}}{8 \pi^{2}}\left[\left(1+\frac{m_{H}^{2}}{s}\right) \log \frac{s}{m_{H}^{2}}-2\left(1-\frac{m_{H}^{2}}{s}\right)\right],
\end{aligned}
$$

where $\beta_{h}^{2}=\left[1-\left(m_{H}+m_{Z}\right)^{2} / s\right]\left[1-\left(m_{H}-m_{Z}\right)^{2} / s\right], v_{e}=-1+4 \sin ^{2} \theta_{W}$ and $a_{e}=-1$. In Fig. 7, we show the Higgs production cross sections as functions of $m_{h}$ in Fig. 7. Depending on the neutral Higgs mixing angle $\alpha$, the predicted cross section can take a wide range compared to the SM predition, especially for low compositeness scale $\Lambda$. For higher $\Lambda$, the allowed $m_{h}$ has a narrow region, since the input parameter $\lambda_{10}$ and the mixing angle $\alpha$ are strongly constrained if the compositeness scale $\Lambda$ is high. Therefore the Higgs production cross section is almost definitely determined as a function of $m_{h}$, when $\Lambda>10^{12} \mathrm{GeV}$. 


\section{Conclusions}

In conclusion, we considered an interesting possibility that the Higgs boson produced at the future colliders is neither a fundamental scalar nor a composite scalar, but a mixed state of them. It could be a generic feature, if there exists a strong dynamics at a high scale which give rise to the dynamical electroweak symmetry breaking, in addition to the usual Higgs mechanism due to the nonvanishing VEV of a fundamental Higgs. It is interesting that this scenario could be easily realized, if we embed the SM lagrangian in a higher dimension with bulk gauge interactions. The bulk gauge interaction can give rise to a new strong dynamics in the 4 dimensional theory and trigger the dynamical symmetry breaking of the electroweak symmetry. We have constructed the simplest model with the NJL type fourfermion interaction of top quarks as the strong dynamics inspired by the BHL and study the phenomenology of the two Higgs doublets model with the compositeness condition as the low energy effective theory. The resulting theory can accommodate the observed top mass, and give specific predictions for neutral and charged Higgs masses at a given value of $\Lambda$. For example, the charged Higgs boson is always lighter than the CP-odd Higgs neutral boson, although the mass difference is very small. For $\Lambda \sim 10^{15} \mathrm{GeV}$, the allowed parameter region gis rather restricted, and we predict $m_{h}>250 \mathrm{GeV}$. Also the charged Higgs boson becomes lighter than $h$, and could be the first signal of our model at the future colliders.

Our study can be extended into various directions. One can consider more general situation where the fundamental Higgs couples both to the bottom and the top quarks. In that case, the resulting low energy effective theory will be a Type-III two-Higgs doublet model. Or one can consider both $t \bar{t}$ and $b \bar{b}$ condense and contribute to the EWSB. Then the resuting theory will be a three-Higgs doublet model with specific matching conditions. Also one has to include all the three generations and construct realistic models with correct CKM

mixing and $\mathrm{CP}$ violation. Some of these issues will be pursued in separate publications in the future.

\section{Acknowledgments}

PK is grateful to Bogdan Dobrescu and Chris Hill for useful discussions. This work is supported in part by KRF Sundo grant R02-2003-000-10085-0, and KOSEF through CHEP at Kyungpook National University (PK), and by Korea Research Foundation Grant KRF2003-050-C00003 (KYL).

\section{Appendix : RG equations}

In this work, we use the 1-loop RG equations for the Type-II Higgs doublet model as given in Ref. [18]. The RG equations for gauge couplings are given by

$$
D g_{i}=-c_{i} g_{i}^{3}
$$


where $D=16 \pi^{2} \partial / \partial \ln \mu$ and

$$
c_{1}=-\frac{1}{6} N_{H}-\frac{20}{9} N_{g}, \quad c_{2}=\frac{22}{3}-\frac{4}{3} N_{g}-\frac{1}{6} N_{H}, \quad c_{3}=11-\frac{4}{3} N_{g},
$$

with the number of generations $N_{g}$ and the number of Higgs doublets $N_{H}$.

The RG equations for Yukawa couplings are given by

$$
\begin{aligned}
D g_{b} & =-\left(8 g_{3}^{2}+\frac{9}{4} g_{2}^{2}+\frac{5}{12} g_{1}^{2}\right) g_{b}+\frac{9}{2} g_{b}^{3}+\frac{1}{2} g_{t}^{2} g_{b}, \\
D g_{t} & =-\left(8 g_{3}^{2}+\frac{9}{4} g_{2}^{2}+\frac{17}{12} g_{1}^{2}\right) g_{t}+\frac{9}{2} g_{t}^{3}+\frac{1}{2} g_{b}^{2} g_{t},
\end{aligned}
$$

and for Higgs quartic self-couplings given by

$$
\begin{aligned}
D \lambda_{1}= & 12 \lambda_{1}^{2}+4 \lambda_{3}^{2}+4 \lambda_{3} \lambda_{4}+2 \lambda_{4}^{2}+2 \lambda_{5}^{2}-3 \lambda_{1}\left(3 g_{2}^{3}+g_{1}^{2}\right)+\frac{3}{2} g_{2}^{4} \\
& +\frac{3}{4}\left(g_{2}^{2}+g_{1}^{2}\right)^{2}+12 \lambda_{2} g_{b}^{2}-12 g_{b}^{4} \\
D \lambda_{2}= & 12 \lambda_{1}^{2}+4 \lambda_{3}^{2}+4 \lambda_{3} \lambda_{4}+2 \lambda_{4}^{2}+2 \lambda_{5}^{2}-3 \lambda_{2}\left(3 g_{2}^{3}+g_{1}^{2}\right)+\frac{3}{2} g_{2}^{4} \\
& +\frac{3}{4}\left(g_{2}^{2}+g_{1}^{2}\right)^{2}+12 \lambda_{1} g_{t}^{2}-12 g_{t}^{4} \\
D \lambda_{3}= & \left(\lambda_{1}+\lambda_{2}\right)\left(6 \lambda_{3}+\lambda_{4}\right)+4 \lambda_{3}^{2}+2 \lambda_{4}^{2}+2 \lambda_{5}^{2}-3 \lambda_{3}\left(3 g_{2}^{2}+g_{1}^{2}\right) \\
& +\frac{9}{4} g_{2}^{4}+\frac{3}{4} g_{1}^{4}-\frac{3}{2} g_{2}^{2} g_{1}^{2}+6 \lambda_{3}\left(g_{b}^{2}+g_{t}^{2}\right)-12 g_{b}^{2} g_{t}^{2} \\
D \lambda_{4}= & \left(\lambda_{1}+\lambda_{2}\right) \lambda_{4}+4\left(2 \lambda_{3}+\lambda_{4}\right) \lambda_{4}+8 \lambda_{5}^{2}-3 \lambda_{4}\left(3 g_{2}^{2}+g_{1}^{2}\right) \\
& +3 g_{1}^{2} g_{2}^{2}+6 \lambda_{4}\left(g_{b}^{2}+g_{t}^{2}\right)+12 g_{b}^{2} g_{t}^{2} \\
D \lambda_{5}= & \lambda_{5}\left[2\left(\lambda_{1}+\lambda_{2}\right)+8 \lambda_{3}+12 \lambda_{4}-3\left(3 g_{2}^{2}+g_{1}^{2}\right)+6\left(g_{b}^{2}+g_{t}^{2}\right)\right] .
\end{aligned}
$$

\section{References}

[1] J. F. Gunion, H. E. Haber, G. Kane and S. Dawson, The Higgs Hunter's Guide, Addison-Wesley 1990.

[2] C. T. Hill and E. T. Simmons, Phys. Rep. 381, 235 (2003)and references therein.

[3] W. A. Bardeen, C. T. Hill and M. Lindner, Phys. Rev. D 41, 1647 (1990); C. T. Hill, Report No. FERMI-CONF-90/170-T.

[4] V. A. Miransky, M. Tanabashi and K. Yamawaki, Phys. Lett. B 221, 177 (1989); Mod. Phys. Lett. A 4, 1043 (1989).

[5] C. T. Hill, Phys. Lett. B 266, 419 (1991).

[6] B. A. Dobrescu and C. T. Hill, Phys. Rev. Lett. 81, 2634 (1998).

[7] B. A. Dobrescu, Phys. Lett. B 461, 99 (1999).

[8] H.-C. Cheng, B. A. Dobrescu and C. T. Hill, Nucl. Phys. B589, 249 (2000).

[9] J. Bardeen, L. N. Cooper and J. R. Schrieffer, Phys. Rev. 106, 162 (1957); Phys. Rev. 108 (1957) 1175. 
[10] D. Bauer, Acta Phys. Pol. B 36, 251 (2005).

[11] M. Luty, Phys. Rev. D 41, 2893 (1990.)

[12] M. Carena, T. E. Clark, C. E. M. Wagner, W. A. Bardeen and K. Sasaki, Nucl. Phys. B 369, 33 (1992).

[13] N. Arkani-Hamed, S. Dimopoulos and G. R. Dvali, Phys. Lett. B 429, 263 (1998); ibid. 436, 257 (1998); L. Randall and R. Sundrum, Phys. Rev. Lett. 83, 3370 (1999); ibid. 83, 4690 (1999); T. Appelquist, H.-C. Cheng and B. A. Dobrescu, Phys. Rev. D 64, 035002 (2001); C. Csaki, C. Grojean, L. Pilo and J. Terning, Phys. Rev. Lett. 92, 101802 (2004); K. Agashe, A. Delgado, M. J. May and R. Sundrum, JHEP 0308, 050 (2003).

[14] H. Georgi, A. K. Grant and G. Hailu, Phys. Lett. B 506, 207 (2001); Phys. Rev. D 63, 064027 (2001).

[15] N. Rius and V. Sanz, Phys. Rev. D 64, 075006 (2001); K. Oda and A. Weiler, Phys. Lett. B 606, 408 (2005).

[16] Work in progress.

[17] S.L. Glashow and S. Weinberg, Phys. Rev. D 15, 1958 (1977).

[18] C. T. Hill, C. N. Leung and S. Rao, Nucl. Phys. B262, 517 (1985).

[19] S. Eidelman et al. [Particle Data Group], Phys. Lett. B 592, 1 (2004).

[20] I.F. Ginzburg and M.V. Vychugin, Presented at the 16th International Workshop on High Energy Physics and Quantum Field Theory (QFTHEP 2001), Moscow, Russia, 6-12 Sep 2001. Published in the proceeding, "Moscow 2001, High energy physics and quantum field theory" p.64-76 hep-ph/0201117; I.F. Ginzburg, M. Krawczyk and P. Osland Presented at International Workshop on Linear Colliders (LCWS 2002), Jeju Island, Korea, 26-30 Aug 2002. Published in "Seogwipo 2002, Linear colliders" p. 90-94 hep-ph/0211371.

[21] M. Moretti, S. Moretti, F. Piccinini, R. Pittau and A. D. Polosa, JHEP 0502, 024 (2005);

S. Kanemura, Y. Okada and E. Senaha, Phys. Lett. B 606, 361 (2005).

[22] S. Kanemura, Y. Okada, E. Senaha and C. P. Yuan, Phys. Rev. D 70, 115002 (2004) [arXiv:hep-ph/0408364].

[23] See, for example, recent reviews and references therein: A. Djouadi, arXiv:hep-ph/0503172; arXiv:hep-ph/0503173. 


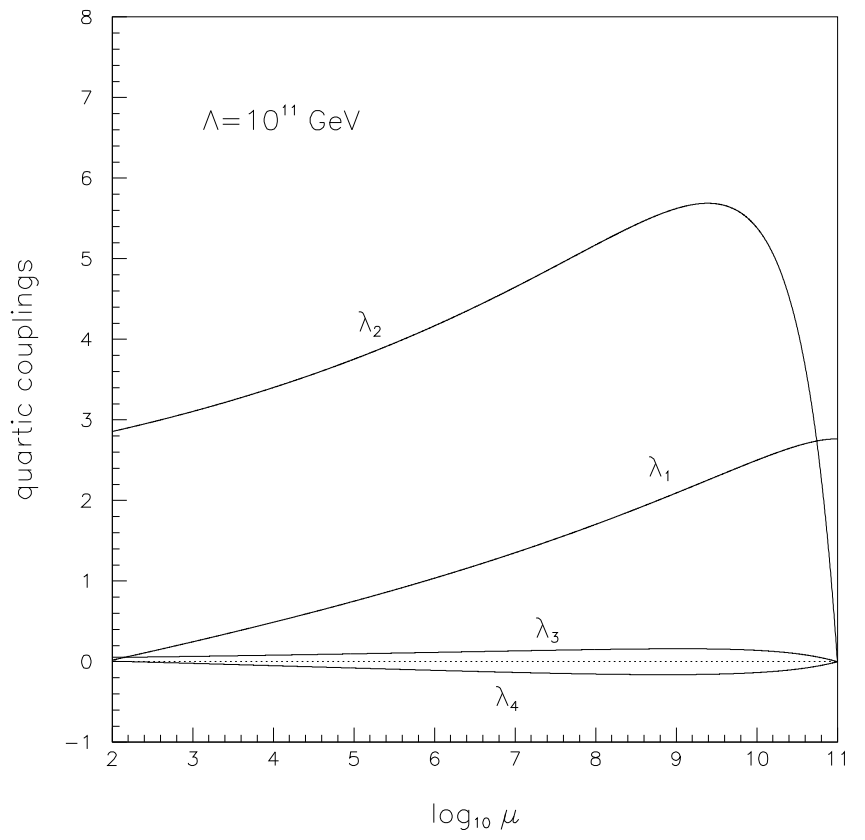

(a)

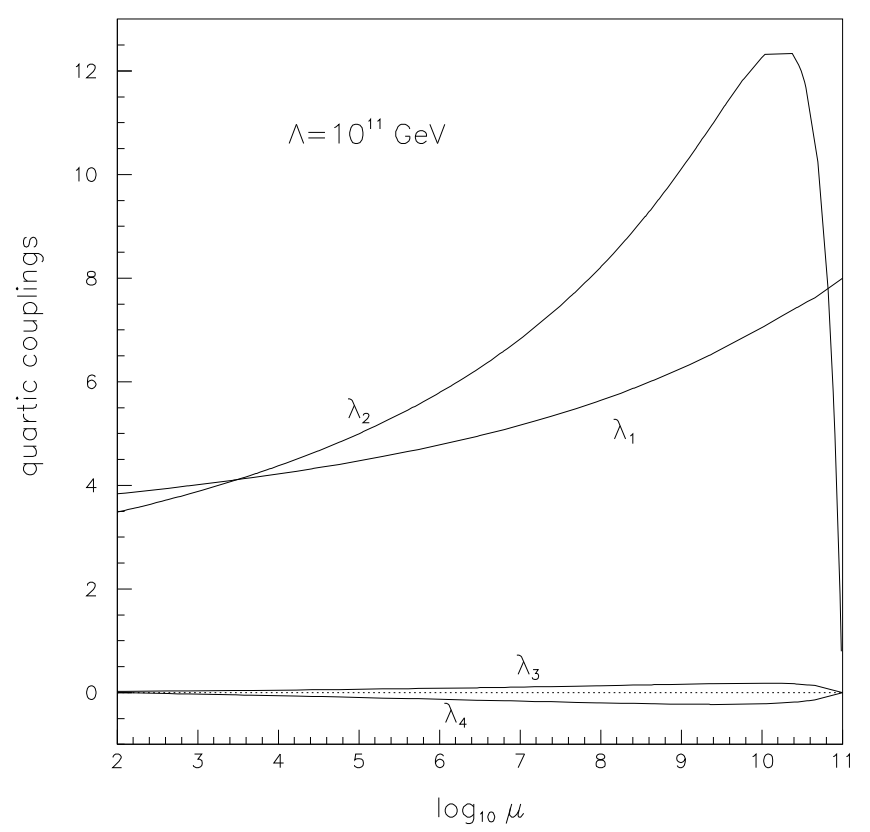

(b)

Figure 2: Evolution of the Higgs quartic coupling $\lambda_{i}$ with respect to $\log _{10} \mu$ for two different $\lambda_{10}$ : (a) and (b) . 


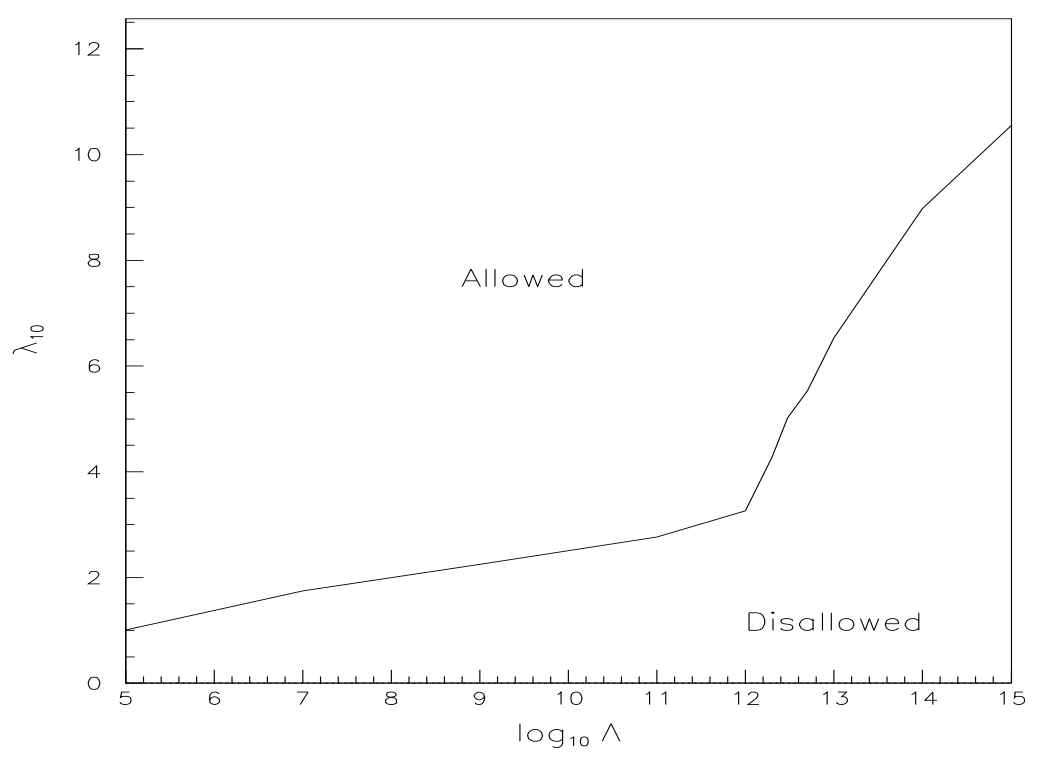

$C$
0
0
0
0
0
0
0
0

Figure 3: Allowed values of the quartic coupling $\lambda_{10}$ at $\Lambda$ with respect to the compositeness scale $\Lambda$. 


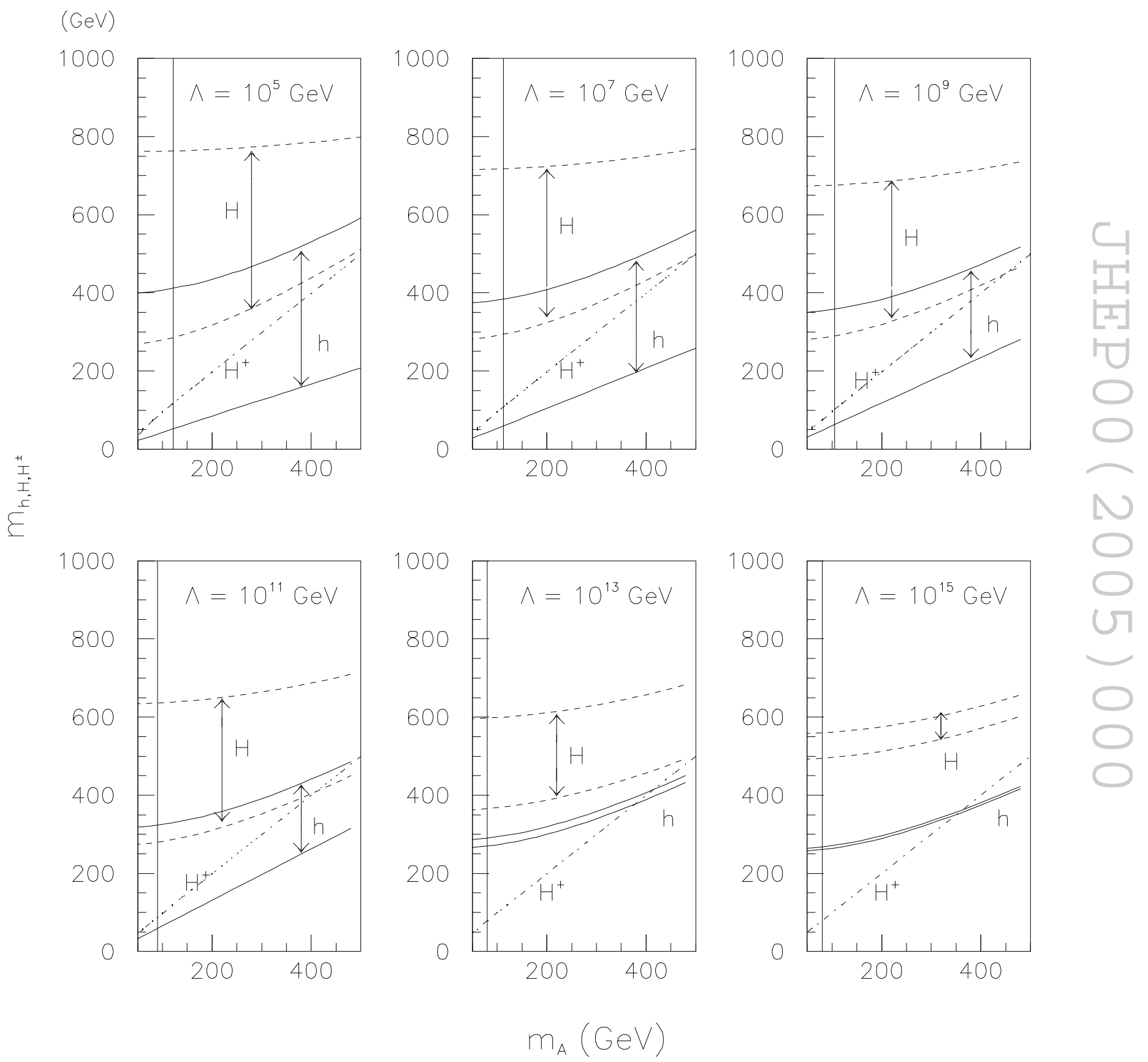

Figure 4: Masses of neutral Higgs bosons $h$ (inside the solid lines), $H$ (inside the dashed lines) and the charged Higgs boson $H^{ \pm}$(dahs-dotted line) with respect to $m_{A}$. 

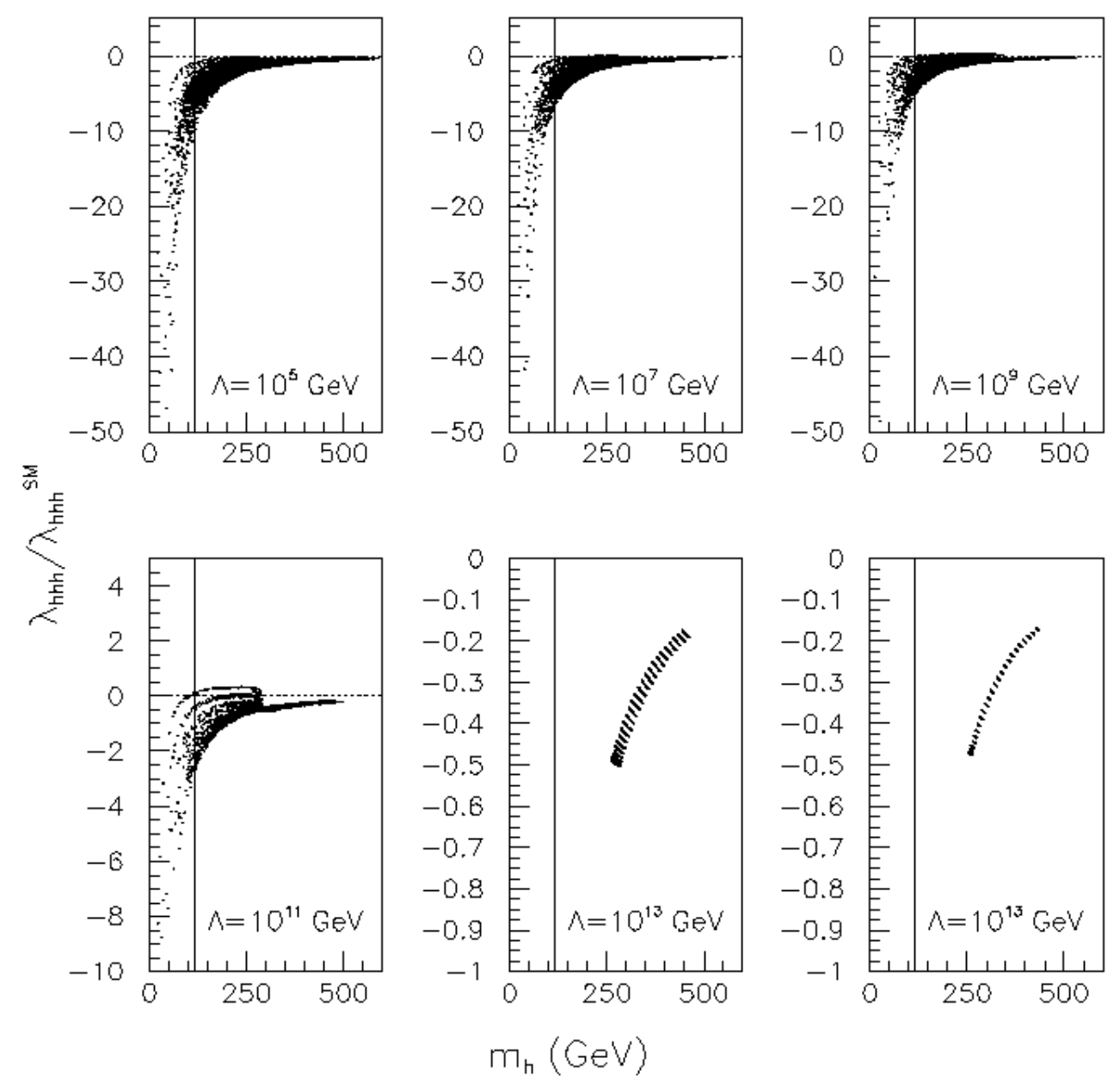

Figure 5: Ratio of our model prediction for the triple self-coupling of the Higgs boson to that of the SM with respect to the lightest neutral Higgs boson mass. The vertical line denotes the lower bound on the Higgs boson mass 

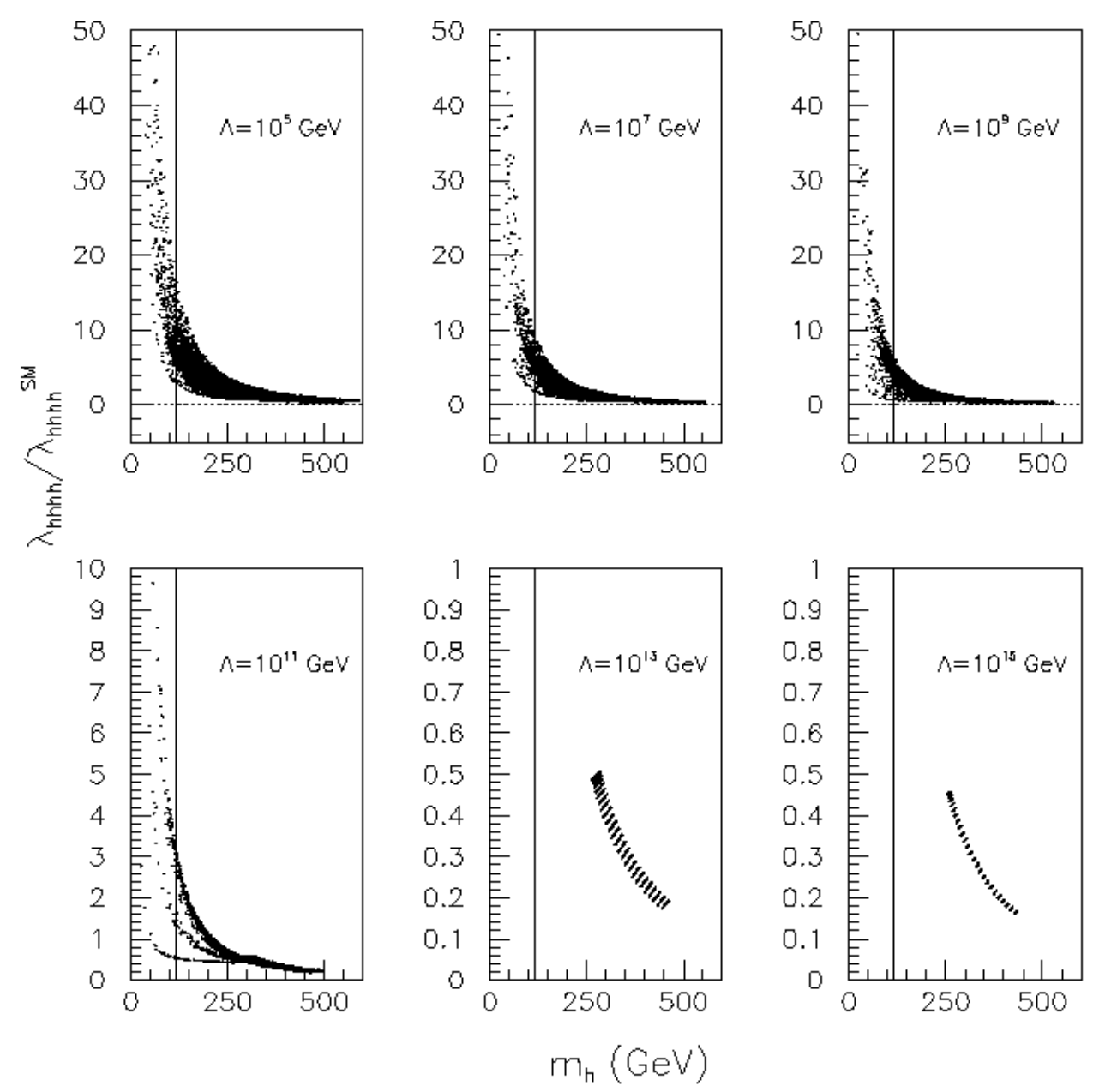

Figure 6: Ratio of our model prediction for the quartic self-coupling of the Higgs boson to that of the SM with respect to the lightest neutral Higgs boson mass. The vertical line denotes the lower bound on the Higgs boson mass 

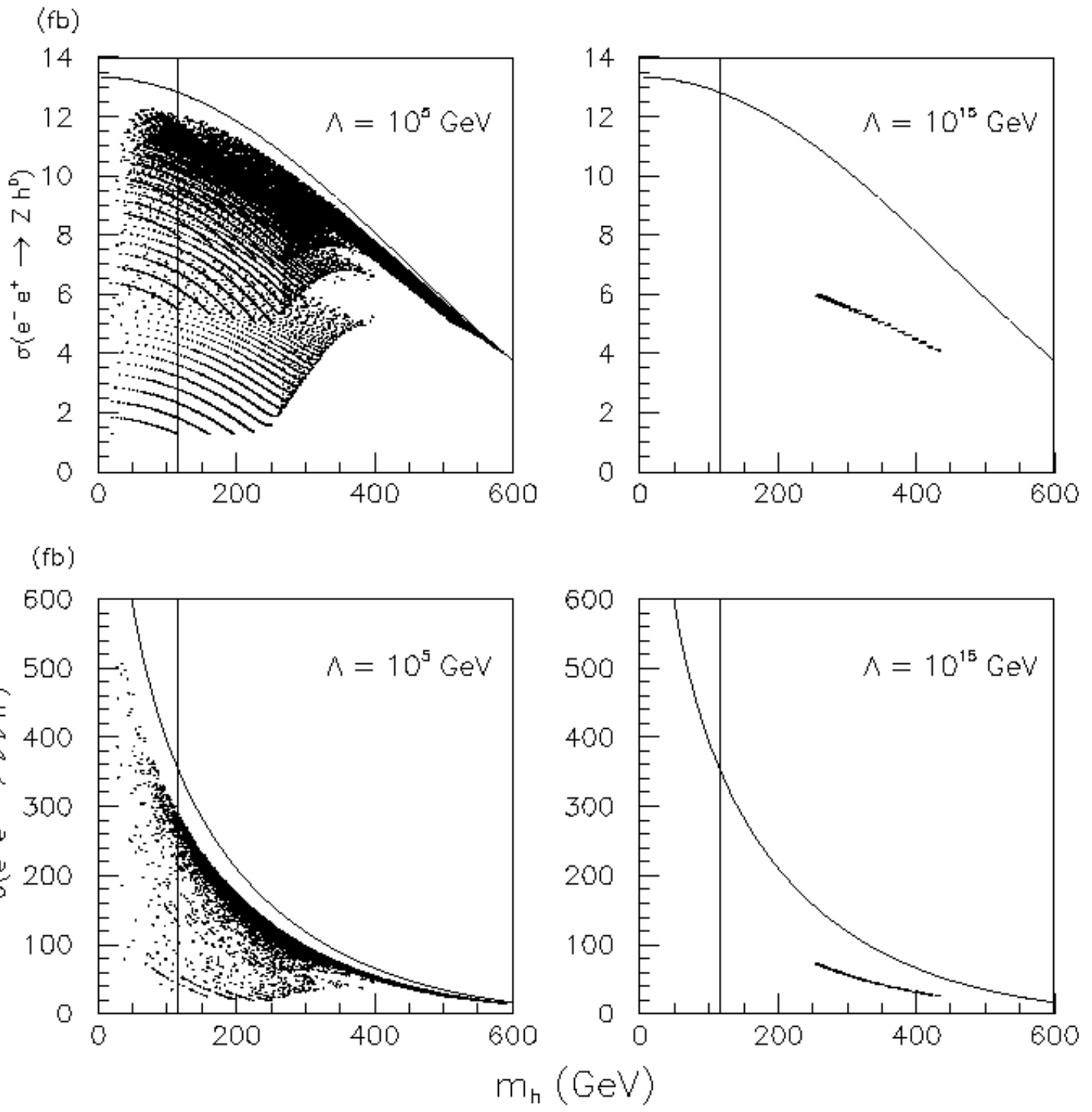

Figure 7: Production cross section of the neutral Higgs boson at the ILC. $\sqrt{s}=1 \mathrm{TeV}$ is assumed. The solid curves denotes the SM predictions. 